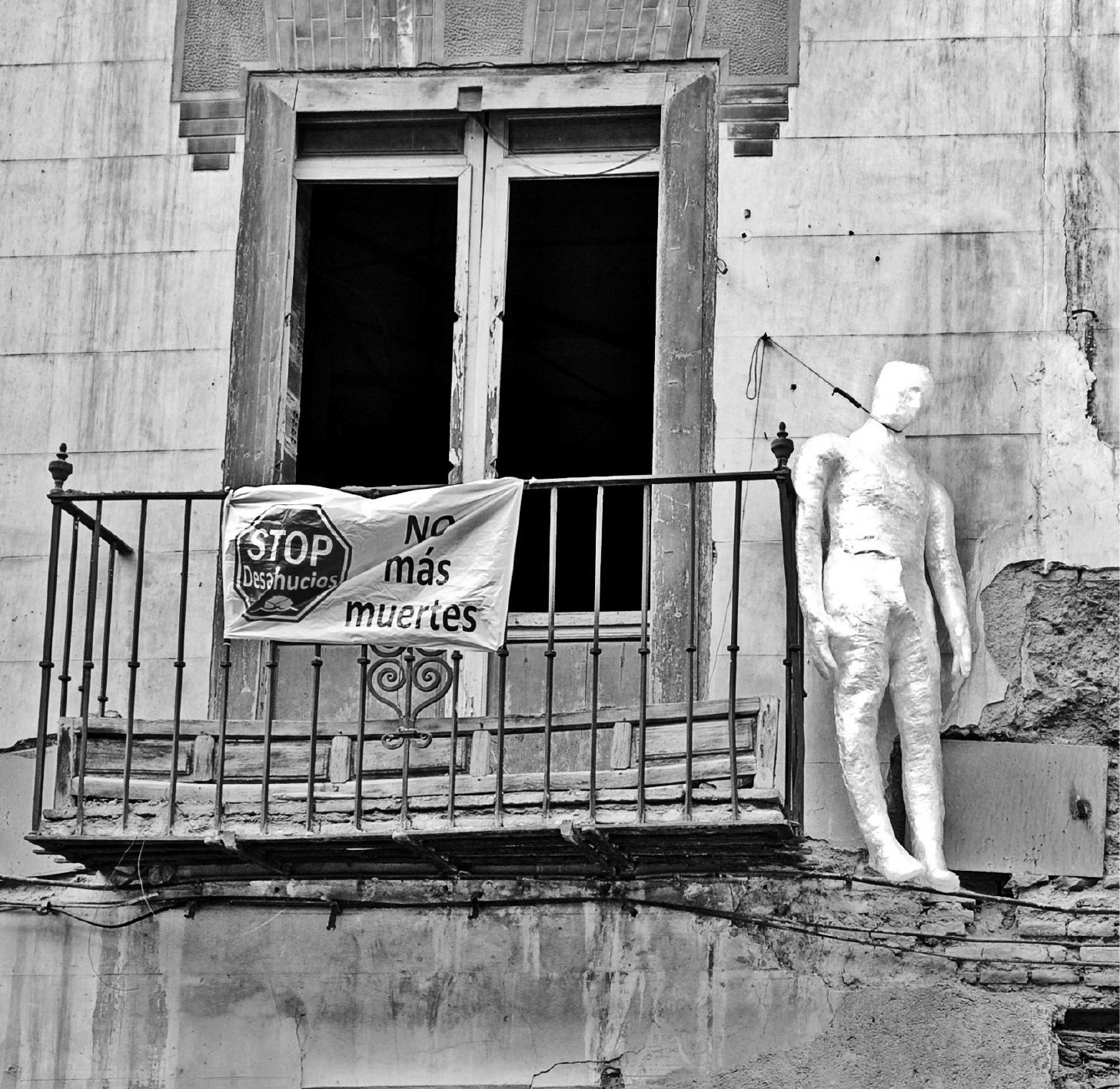

AIBR

Revista de Antropología

Iberoamericana

www.aibr.org

Volumen 15

Número 2

Mayo - Agosto 2020

Pp. 247 - 271

Madrid: Antropólogos

Iberoamericanos en Red.

ISSN: 1695-9752

E-ISSN: 1578-9705
Habitar la investigación en la universidad neoliberal

y eurocentrada: La etnografía colaborativa

como apuesta por lo común y la subjetivación política

Aurora Álvarez Veinguer

Departamento de Antropología Social, Facultad de Filosofía y Letras de la Universidad de Granada.

Luca Sebastiani

Centro de Estudos Sociais (CES), Universidade de Coimbra.

Recibido: 02.03.2018

Aceptado: 10.12 .2018

Dol: 10.11156/aibr.150204 


\title{
RESUMEN
}

En este artículo, primero recorremos las transformaciones que han afectado a la institución académica a nivel global y europeo, concluyendo que la Universidad actual es neoliberal y eurocentrada. Resaltamos los cambios acontecidos en la investigación — que se vuelve cada vez más «rápida», individualista y despolitizada-, en los procesos de subjetivación del personal investigador — que constantemente necesita acumular méritos curriculares y alcanzar «excelencia» e «impacto»- y señalamos la precarización de las condiciones laborales y de vida. Posteriormente, proponemos posibles líneas de fuga a partir de nuestra experiencia de etnografía colaborativa junto con «Stop Desahucios Granada-15M». A través de los ejemplos discutidos, argumentamos que esta práctica de campo puede ayudar a descolonizar las formas de hacer investigación y resaltamos su potencial para producir saberes colectivos e impulsar procesos de subjetivación política. Concluimos que la investigación colaborativa constituye un potente revulsivo para contrarrestar las lógicas individualistas y despolitizadoras hegemónicas.

PALABRAS CLAVE

Universidad neoliberal, descolonialidad, etnografía colaborativa, saber(es) común(es), subjetivación política.

\section{INHABITING RESEARCH IN THE NEOLIBERAL AND EUROCENTRIC UNIVERSITY: COLLABORATIVE ETHNOGRAPHY AS A BET FOR THE COMMON AND POLITICAL SUBJECTIVATION}

\begin{abstract}
In this paper, we discuss the transformations undergone by the academic institution at global and European level, arguing that contemporary University is both neoliberal and eurocentric. First, we highlight the changes that have affected research practice, conceived as an increasingly "fast", individualist and depoliticized activity; the role (and the subjectivity) of the researcher, constantly pushed to accumulate academic merits and achieve "excellence" and "impact"; and the growing precaritization of both working and life conditions. Then, drawing on our experience of collaborative ethnography with "Stop Evictions Granada-15M" (Spain), we propose possible "lines of flight" from this situation. Based on our fieldwork, we theorize collaborative ethnography as a way to decolonize research practices. We stress its potential to produce collective knowledge and promote (re)politicization processes and claim that collaboration can help overcome the (currently hegemonic) individualistic and depoliticizing logics in Academia.
\end{abstract}

\section{KEY WORDS}

Neoliberal University, decoloniality, collaborative ethnography, collective knowledge, (re) politicization. 


\section{Introducción}

Este artículo tiene dos objetivos: primero, queremos esbozar las principales problemáticas que afectan a la producción de conocimiento en la Universidad contemporánea, centrándonos en las prácticas de investigación más situadas, implicadas y activistas. Luego, discutiremos posibles maneras de sortear dichas dificultades, a partir de una experiencia de campo concreta en la que un grupo de personas estamos comprometidas desde diciembre de $2015^{1}$ : se trata del proceso de coinvestigación junto a «Stop Desahucios Granada-15M» (contexto español), un colectivo que lucha por el derecho a la vivienda parando desahucios, apoyando a familias endeudadas por causas hipotecarias o problemas de alquiler, y forzando a entidades bancarias e instituciones a negociar e impulsar cambios legislativos ${ }^{2}$. Aportando ejemplos de esta experiencia, mostraremos cómo estamos tratando de "atravesar» el complejo escenario que nos rodea, buscando líneas de fuga de los condicionamientos de la institución académica actual — con un particular enfoque desde la antropología social—. Todo esto con dos salvedades: primero, nuestra crítica a la Universidad neoliberal se articula desde un posicionamiento interno a ella: no ha de leerse, por tanto, como una condena moralista, sino como un cuestionamiento planteado desde la práctica de la reflexividad epistemológica-política a partir de nosotras mismas ${ }^{3}$. Segundo: no pretendemos aportar soluciones universales, sino, por el contrario, queremos contribuir con reflexiones encarnadas a partir de nuestro contexto concreto.

Primero, discutiremos las tendencias de la Universidad actual —que definiremos neoliberal y eurocentrada-, siendo el contexto-institución donde trabajamos. Después de mapear algunas de las tensiones que la atraviesan, conceptualizaremos la etnografía colaborativa —que también llamamos co-labor - enunciando algunos de los posibles ejes, andamiajes,

1. La investigación forma parte del proyecto «Procesos emergentes y agencias del común: praxis de la investigación social colaborativa y nuevas formas de subjetivación política», Proyectos de I+D+i (Convocatoria 2014) del programa estatal de fomento de la investigación científica y técnica de excelencia, Ministerio de Economía y Competitividad del Gobierno de España (Referencia: CSO2014-56960-P). Aunque solo dos personas firmen el artículo, este se nutre de un proceso de coinvestigación colectivo junto al resto del equipo, formado por: Antonia Olmos Alcaraz, Ariana Sánchez Cota, Rocío García Soto y Borja Íñigo Fernández Alberdi, así como por muchos/as compañeros/as de Stop Desahucios-15M Granada (https://afectadosporlahipotecagranada.com/) de las Asambleas Zaidín y Centro.

2. El acceso a una vivienda digna es un problema estructural en el contexto español, originado por el enfoque propietario y especulativo adoptado por las administraciones públicas desde hace décadas; sin embargo, se ha intensificado desde el inicio de la crisis de 2007. 3. Y sin perder de vista nuestra condición relativamente privilegiada, encarnada por cada cual desde su intersección entre clase, género, «raza»/etnicidad y otras posicionalidades. 
entendiéndolos como una tentativa para descolonizar la investigación social. A continuación, dedicaremos una especial atención a dos de los cuatro ejes propuestos — superar el modelo de investigación individualista y los saberes despolitizados alentados por la Universidad neoliberal—, además de mencionar la relevancia de colocar en el centro los cuidados y las emociones y la propuesta de desplegar pluriversos metodológicos. Pensamos que la etnografía colaborativa puede aportar mucho respecto a todos los anteriores ejes, si bien, por razones de espacio, nos centraremos en los dos primeros. En una última sección, concretaremos la discusión aportando ejemplos desde nuestra coinvestigación y presentaremos algunos de los desbordes que hemos intentado practicar, sorteando las tendencias más evidentes de la Universidad neoliberal y visibilizando las respuestas que vamos dándole desde la etnografía colaborativa. Finalmente, concluiremos recapitulando las cuestiones principales.

\section{Desde dónde escribimos: la Universidad neoliberal y eurocentrada}

«Universidad neoliberal», «Universidad empresa» o «Capitalismo académico» son solo algunos de los términos generalmente utilizados para describir los cambios que han afectado a la institución universitaria en las tres últimas décadas. Todas estas expresiones remiten a un cambio central para dicha institución: "[...] su incorporación a los circuitos empresariales y mercantiles de la sociedad capitalista actual» (Galcerán, 2010: 15). El proceso comenzó a finales de los setenta y se intensificó dos décadas después: en el contexto de la "economía global del conocimiento", este último pasó de ser considerado un factor instrumental para el desarrollo económico a convertirse en elemento central dentro de las lógicas de valorización capitalista. Dicho con otras palabras, el conocimiento se estaba convirtiendo en una mercancía (Wright y Rabo, 2010: 2-3). Este proceso, lejos de ser inevitable, se impuso mediante reformas institucionales e intervenciones legislativas determinadas (Lander, 2005: 37-38) ${ }^{4}$. Así, se redujeron los fondos públicos para la enseñanza y la investigación (Shore, 2010: 15) —favoreciendo la penetración de intereses privados y comerciales-y se aprobaron leyes restrictivas en materia de propiedad intelectual y patentes, limitando el acceso al conocimiento. Estas reformas per-

4. La misma noción «sociedad del conocimiento", transmitiendo un imaginario tecnocrático y pacificado de "gestión técnica del cambio", facilitó la naturalización de la toma de ciertas decisiones políticas (Serrano y Crespo, 2002: 192). 
mitieron la implantación de un «mercado global de la educación», tal como requería el Banco Mundial (Galcerán, 2010: 20).

Ante el liderazgo del proceso por parte de universidades estadounidenses, británicas y australianas (Wright y Rabo, 2010: 4), numerosos gobiernos europeos firmaron la declaración de Bolonia (1999), que inició una cooperación intergubernamental con vistas a la armonización de las legislaciones nacionales en materia de enseñanza y estableció el Espacio Europeo de Educación Superior — seguido un año después del Espacio Europeo de Investigación-. El objetivo declarado de estas medidas era "[...] preparar el paso a una economía y una sociedad basadas en el conocimiento» (Consejo Europeo, 2000). En el contexto español —desde donde escribimos - la Universidad neoliberal fue implantada mediante la Ley Orgánica de Universidades (L.O. 6/2001) y sus posteriores reformas, que transformaron el sistema de enseñanza secundaria y fragmentaron las condiciones del personal docente, investigador y de administración y servicios $^{5}$. Estas disposiciones, aun caracterizándose por una retórica de «apertura a la sociedad», la entendían en los términos de una "empresa» o un «mercado» (Montalba Ocaña, 2015: 93), impulsando "[...] una comprensión de la ciencia exclusivamente en términos de beneficio, entendido en términos macroeconómicos, lo que advierte un tipo de reduccionismo científico donde la economía -y una economía de cierto tipo-es la que al final tiene los criterios de valoración de la empresa científica» (Eizagirre, 2016: 825).

Estas transformaciones se enmarcaban en la emergencia de la «nueva gestión pública», esto es, la extensión a la administración estatal de formas organizativas y lógicas de gestión empresariales. Desde una racionalidad mercantil, se ensalzaron los valores de la competición, rentabilidad y eficacia financiera. Para su implementación, se desplegaron prácticas de «gobierno a través de los números» (Shore y Wright, 2015) orientadas a medir, evaluar y monitorear la actuación de los múltiples agentes implicados en la administración de lo social y a determinar los criterios para su funcionamiento «óptimo». Era el triunfo de la "cultura de la auditoría». En su traslación a la Academia, este régimen de prácticas se materializó en la proliferación de indicadores, ranking, evaluaciones comparativas y otras prácticas de cálculo (Shore, 2010: 15-16) enfocadas a medir el

5. Las reformas encontraron resistencia por parte de movimientos estudiantiles, aunque consiguieron seducir a sectores del personal docente e investigador mediante la reapropiación instrumental de críticas sobre la autorreferencialidad, burocracia y endogamia de la institución académica — la famosa metáfora de la «torre de marfil»— originariamente emergidas en los movimientos estudiantiles de décadas anteriores (Gómez y Jódar, 2013: 91; Risager y Thorup, 2016: 12). 
«rendimiento académico» y a estimular su mejora mediante la competición. Desde esta perspectiva, ya "[n]o es necesario privatizar las universidades, solo es preciso que estas funcionen como empresas» (Ferreiro Baamonde, 2010: 120). Nuevos «saberes expertos» —como las disciplinas bibliométricas - y nuevos actores —agencias evaluadoras de todos tipos- aparecieron en este escenario (Shore, 2010). Queremos resaltar tres consecuencias, acarreadas por estas transformaciones, en los modelos de investigación, los procesos de subjetivación del personal investigador y su condición laboral-existencial:

- La implantación de la Universidad neoliberal ha acelerado profundamente los tiempos de la investigación (y la docencia), privilegiando estudios "cortoplacistas", rentabilizables en tiempos breves y con un impacto fácilmente medible (Escobar, 2007: 55; Greenwood, 2012: 118). Para las nuevas lógicas productivistas, el tiempo es un capital que no puede ser derrochado: así pues, el modelo de Universidad humboldtiana (Risager y Thorup, 2016: 20), centrado en la investigación «básica» y no influido por la urgencia de alcanzar resultados a breve plazo, ha sido reemplazado por un paradigma en donde los productos científicos son subordinados a «[...] criterios y ranking que valoran principalmente lo cuantificable, exhibible y comercializable» (Colectivo Indocentia, 2016). En este contexto, se impulsan ritmos vertiginosos de producción de artículos y comunicaciones, facilitando la redacción de textos «refritos» y "cuasi-fotocopia» el uno del otro (Díez Gutiérrez, 2016) y el recurso a «astucias y triquiñuelas» de todo tipo — desde el falseamiento de resultados en las ciencias «duras», al exceso de «autocitas», «autoplagios», "redes de citas», "guerras de citas», etc. (Colectivo Indocentia, 2016; Díez Gutiérrez, 2016)— ${ }^{6}$. Se hace creciente la cantidad de "papeles», "certificados» $y$ «formularios» que hay que firmar, rellenar y acumular para acreditar méritos, acceder a convocatorias o conseguir evaluaciones positivas: pese a la retórica antiburocrática que caracteriza los discursos de la nueva gestión pública, la burocracia no es reducida: más bien, la Universidad neoliberal redibuja sus funciones y la pone al servicio de sus lógicas evaluadoras y prácticas de cálculo, sin por eso hacerla más trans-

6. Otro ejemplo de estas tendencias es lo que podríamos llamar «proyectitis»: dada la creciente falta de recursos públicos y la consecuente necesidad de encontrar subvenciones participando en convocatorias nacionales/europeas/internacionales complejas y extremadamente competitivas, se multiplica la redacción de proyectos ad hoc, de manera serial y continuada (Blommaert, 2015; Migliaccio, 2016). Vista la escasa tasa de éxito (a veces inferior al $1 \%$ ), se han levantado dudas sobre el derroche de energías, tiempo y dinero que esto supone, cuando dichos recursos podrían ser dedicados directamente a la investigación básica (Blommaert, 2015). 
parente (Alonso y Fernández Rodríguez, 2016; Barnés, 2015; Chomsky, 2013; Irigoyen, 2011: 41; Shore, 2010).

- Se han difundido y normalizado nociones como "excelencia», "calidad», "responsabilidad», "espíritu emprendedor», entendidas como metas a alcanzar por el personal investigador. Estos conceptos poseen cierta polisemia seductora, remitiendo a significados distintos según quien los pronuncie (Wright y Rabo, 2010: 6). Sin embargo, en el sentido hegemónico que se ha venido configurando, apuntan básicamente al impacto bibliométrico de un producto científico — que "[s]olo será excelente si es citado infinidad de veces» (Alinovi, 2014: 12) — o a la capacidad de una investigación para generar riqueza económica a breve plazo (Shore, 2010: 15-16). Más allá de los matices semánticos, cabe señalar lo que estos conceptos «[...] permiten hacer» (Colectivo Indocentia, 2016): antes que nada, su normalización ayuda a naturalizar la "necesidad de competir» (Colectivo Indocentia, 2016). Esto ha contribuido a moldear fuertemente las subjetividades investigadoras, contribuyendo a producir "[...] un sujeto que interioriza que la evaluación permanente le es la condición para la realización de su proyecto» (Irigoyen, 2011: 42). En el caso de la Universidad española, la mayoría de los procedimientos de evaluación son de tipo voluntario ${ }^{7}$ : nadie está obligado a someterse a ellos, pero (casi) todos lo hacen, siendo la única manera para conseguir acreditaciones, incrementos retributivos y mejores condiciones laborales (Gómez y Jódar, 2013). Así pues, "[1]as nuevas formas de gobierno neoliberal modifican nuestras subjetividades: nos convertimos en investigadores competitivos, activos, polivalentes y flexibles, en 'empresarios de nosotros mismos'. El 'profesorado excelente' trabaja solo por y para sí. Incorporamos el cálculo coste-beneficio no solo a la hora de planificar una investigación, sino también a la hora de gestionar nuestras relaciones» (Colectivo Indocentia, 2016). Todo esto genera un creciente «resentimiento» (Galcerán, 2010: 18), «rivalidades» (Montalba Ocaña, 2015; Shore, 2010: 25), fomenta una cultura «de la desconfianza» (Halffman y Radder, 2015: 167) y «del rendimiento" (Shore, 2010: 27) y una situación de "ansiedad crónica» y «estrés» (Gill, 2015: 50).

- La hiperproductividad científica termina invadiendo y difuminando la frontera entre tiempo de ocio y horario laboral (Colectivo Indocentia, 2016; Gómez y Jódar, 2013: 92-93). Esto es la consecuencia de cambios más trascendentes relacionados con la sociedad posfordista, particular-

7. Evidentemente, se trata de una «voluntariedad» muy relativa, pues los constreñimientos generados por el no sometimiento a los procesos evaluadores terminan reduciendo, cuando no bloqueando, las posibilidades de avanzar en la carrera o incluso estabilizar las propias condiciones de trabajo — sobre todo en el caso del personal investigador más joven-. 
mente acentuados en el contexto del «trabajo cognitivo» (Berardi, 2003: 16) del que la Universidad es expresión paradigmática. Sin pretender abordar una discusión general, resaltamos que «[...] uno de los elementos más reseñables de los nuevos escenarios es la precarización del trabajo y del empleo, que se ha estirado hasta afectar a la totalidad de la vida [...] difuminando las fronteras nitidas que separaban los ciclos formativos del desempeño de la actividad laboral, el tiempo de trabajo del no-trabajo o el consumo de la producción» (Lara y Álvarez, 2009: 109-112). La inseguridad e inestabilidad generalizadas nos permiten hablar de la precariedad como condición de vida, que atraviesa tanto la esfera «(re)productiva» como los procesos de subjetivación de los individuos. Este paradigma ha repercutido con fuerza en la materialidad de las prácticas investigadoras (y docentes): para limitarnos a la Universidad española, se resalta un recurso «excesivo» a contratos precarios, por encima del umbral legalmente previsto del 40\% (Pérez Rastrilla, 2017). Un reciente estudio realizado en la Universidad Complutense de Madrid apunta a un escenario preocupante de precariedad generalizada, caracterizado por salarios bajos con pocas garantías, una carga laboral exagerada y la extrema fragilidad — cuando no inexistencia- de perspectivas estables de trabajo y de vida para los jóvenes académicos (Castillo y Moré, 2017). Todo esto termina influyendo en las formas, los tiempos y hasta los diseños concretos de los proyectos implementados, empujándonos a resaltar el devenir precario de la investigación misma.

Llegados a este punto, aclaramos que la Universidad anteriormente existente no constituye para nosotras un modelo idealizado de referencia. Lejos de nostalgias románticas, reconocemos que la institución universitaria siempre ha jugado un papel crucial para la reproducción del sistema social (Galcerán, 2010: 14) naturalizando, impulsando y legitimando saberes y poderes hegemónicos — sin perjuicio de las numerosas y loables excepciones-. En particular, el que tuviera un carácter "público» y «estatal» no ha implicado que estuviera al servicio de los bienes colectivos ni de lo(s) «común(es)», sino que, más bien, ha supuesto su identificación con el Estado-nación —institución central del sistema mundo moderno/ colonial—, sus retóricas de inclusión/exclusión y sus intereses. Es más, la institución universitaria moderna constituyó desde sus comienzos una punta de lanza crucial para el triunfo del proyecto de Modernidad eurocentrada, y fue conformándose como un «templo del Saber», una institución generadora de verdades pretendidamente universales (Escobar, 2007: 51), enunciadas desde la «hybris del punto cero» neutral y desinteresado (Castro-Gómez, 2005) y válidas en todo tiempo y lugar. Llegó a ser el espacio privilegiado para el «descubrimiento» y la difusión de conoci- 
mientos científicos, racionales y objetivos (Lander, 2005: 4), a la vez que otros saberes eran deslegitimados como «míticos», «irracionales» o «emotivos» y relegados a una posición de no-verdad, ya se tratara de conocimientos experienciales atribuidos al "pueblo llano»—-tachados de meras «supersticiones»- o de las cosmovisiones de pueblos indígenas considerados ajenos a la civilización europea y a su «misión» —lamentablemente, la antropología no ha sido ajena a este proceso-. Para limitarnos a las ciencias sociales, estas han sido eurocéntricas desde la constitución de sus disciplinas, no solo por haberse originado en países occidentales como Francia, Gran Bretaña, Alemania, Italia y los Estados Unidos (Wallerstein, 1997: 97), sino porque «[...] surgieron como respuesta a problemas europeos en un momento de la historia en el que Europa dominaba todo el sistema-mundo» (Wallerstein, 1997: 98). En definitiva, la institución universitaria siempre ha estado vertebrada por la «colonialidad del saber», siendo esta una dimensión central del proyecto moderno-colonial que comenzó a cimentarse en el siglo XV (Castro-Gómez, 2005; Lander, 2000). A esto hay que añadirle las recientes transformaciones en un sentido neoliberal, y es a partir del conjunto de estas articulaciones entre colonialidad del saber y neoliberalismo que desciende nuestra urgencia no solamente por cuestionar los saberes mercantilizados y competitivos de la "cultura de la auditoría", sino también por impugnar el conjunto de las jerarquías epistemológicas y los silenciamientos históricos, abogando por un proceso más amplio de descolonización de los saberes que no puede sino empezar por la(s) manera(s) en que estos son generados.

\section{Descolonizar la investigación: la etnografía colaborativa como co-labor}

En las últimas décadas se viene debatiendo sobre el giro decolonial y la necesidad de descolonizar las ciencias sociales, realizando una apuesta por superar el principio extractivo que las ha caracterizado y dedicando especial atención a las dimensiones epistemológicas y teóricas. Preguntarnos para qué, para quién y cómo producimos conocimiento desde las ciencias sociales invita a prestar atención a la colonialidad del saber y a contrastar la tendencia histórica de las ciencias sociales a invisibilizar los lugares de enunciación. La crítica al canon cientificista (neutral y objetivo) ha sido extensamente debatida especialmente desde los contextos feministas, reivindicando la centralidad de los «saberes situados» (Haraway, 1991), la «política de la localización» (Rich, 1986) o el «incardinamiento como posicionalidad»(Braidotti, 2004). Desde el contexto de la antropología 
social, en 1971 Rodolfo Stavenhagen escribía ya sobre cómo descolonizar las ciencias sociales y planteaba la necesidad de hablar de "observación activista» u «observación militante» (1971: 51), frente a la clásica concepción de "observación participante». Sin duda, la corpopolítica del conocimiento que se viene reivindicando desde el enfoque decolonial (AAVV, 2015; Castro-Gómez y Grosfoguel, 2007; Leyva, Burguete y Speed, 2008; Lugones, 2008; Smith,1999; Walsh, Schiwy y Castro-Gómez, 2002), así como la propuesta de Boaventura de Sousa Santos de las epistemologías del Sur (2009), nos permiten repensar los cimientos que han sustentado el proyecto cientificista universal para tratar de abandonar el «extractivismo epistemológico» (Grosfoguel, 2016) y caminar hacia un cuestionamiento metodológico-político de las formas de investigar.

Desde nuestra experiencia, entendemos la etnografía colaborativa o de co-labor como una de las posibles maneras de aterrizar, desde la práctica concreta, otras maneras de habitar la investigación ${ }^{8}$. ¿Cuáles son algunas de sus principales aportaciones? Implica reconocer otros sabereshaceres, buscar otras formas que incorporen la centralidad del grupo frente al individuo como protagonista de la investigación (coinvestigación), protagonistas colectivos que puedan desplegar espacios afectivos de escucha y diálogos (superando la eterna dicotomía «racionalidad-sentimientos») y así traspasar las dinámicas monológicas sustentadas en la autoridad única y exclusiva del "saber experto", negando otras maneras posibles de explicar y (re)presentar el día a día de la investigación. Tratando de contestar a la pregunta: "¿en un mundo saturado de relaciones coloniales, hasta qué punto es posible un método de investigación descolonizado?» (Hale, 2011: 493), presentaremos algunos de los pasos que estamos dando en un intento particular de descolonizar la investigación ${ }^{9}$. Sin pretender responderla en términos absolutos, sino a partir de

8. Hablamos de «habitar la investigación» porque nos situamos desde un contexto de investigación que nos interpela y nos atraviesa, una «etnografía corporeizada» (Esteban, 2004).

9. Desde 2009, un grupo de personas vinculadas a la Universidad de Granada hemos organizado seminarios, jornadas y conversatorios sobre la mirada decolonial en el contexto universitario. Se organizaron las jornadas «Diálogos entre Ciencias Sociales y Movimientos Sociales. Miradas, Preguntas, (Des)encuentros», financiadas por el Ministerio de Ciencia e Innovación (CSO2009-07790-E), el Plan Propio de Investigación de la Universidad de Granada (2009) y la Consejería de Innovación, Ciencia y Empresa de la Junta de Andalucía (2009). En marzo de 2011 organizamos el taller «Herramientas para las metodologías participativas: usos, aplicaciones y estrategias», en el cual participaron más de una quincena de colectivos y asociaciones de la ciudad de Granada. Fruto de todo este proceso colaborativo, en 2012 publicamos el libro Tentativas, Contagios, Desbordes. Territorios del Pensamiento, publicado por la Universidad de Granada (https://www.researchgate.net/publication /267510017_Tentativas_contagios_desbordes_Territorios_del_pensamiento). En marzo de 
los procesos de investigación que venimos habitando, aportamos cuatro posibles andamiajes para la etnografía colaborativa: i) superar el modelo etnográfico individual activando practicas investigadoras de lo(s) común(es); ii) entender la subjetivación política como parte del proceso de coinvestigación; iii) colocar en el centro los cuidados y las emociones y iv) desplegar pluriversos metodológicos, reformulando y desbordando los métodos de investigación. Puesto que en el apartado posterior abordaremos los dos primeros ejes, en las siguientes líneas presentaremos algunas reflexiones sobre el tercero y el cuarto ${ }^{10}$.

- Desde los trabajos autoetnográficos de numerosas feministas (Behar, 1996; Gregorio Gil, 2006; Lutz y Abu-Lughod, 1990) hemos aprendido la necesidad de incorporar los sentimientos, las emociones, los afectos y los cuidados en nuestras investigaciones. Asumimos que la etnografía colaborativa es un trabajo evidentemente situado y encarnado que constituye un ecosistema de relaciones afectivas, donde las vulnerabilidades de nuestros cuerpos políticos (Scheper-Hughes y Lock, 1987) las pensamos como potencialidades que pueden actuar como cajas de resonancia dentro y fuera del grupo. En nuestro caso, además, son los propios procesos micropolíticos de Stop Desahucios Granada-15M que nos llevan a ello. En el colectivo, las prácticas de cuidado colectivo tienen una importancia relevante: desde que una persona acude al grupo, tanto la asesoría colectiva llevada a cabo en las asambleas como los grupos de apoyo más reducidos constituidos para cada caso — ayudando en la tramitación de papeles, la negociación con los bancos, organizando acciones de protesta, etc.- se dirigen hacia una "politización del sufrimiento» (FernándezSavater, 2008). El estigma por haber perdido la vivienda es reconvertido en un asunto político, los sentimientos individuales de «fracaso» inicialmente experimentados son relativizados, y a veces se genera un autoempoderamiento mediante la lucha social. Este ejemplo rescata la centralidad política de sentimientos y emociones y sustenta nuestra apuesta por llevar a cabo un análisis «somático y vulnerable» (Esteban, 2015).

- En este proceso estamos aprendiendo que los interrogantes de investigación se plantean a partir de las vivencias diarias junto al colectivo. Por eso, hemos desplegado formas dialógicas y horizontales de escucha, generando dispositivos de co-labor creativos y plurales. Ante las me-

2014 organizamos desde la Red Saberes Instituyentes, y gracias al apoyo del Departamento de Antropología Social, el Departamento de Historia Contemporánea, la Facultad de Filosofía y Letras y el Instituto de Migraciones de la Universidad de Granada, un conversatorio sobre la descolonización del conocimiento (http://antropologia.ugr.es/pages/tablon/*/noti cias/conversatorio-la-descolonizacion-del-conocimiento-dialogos-transdisciplinares). 10. Hemos tratado con más profundidad el tema de los cuidados-emociones y el pluriversalismo metodológico en Olmos Alcaraz, Cota, Álvarez Veinguer y Sebastiani (2018). 
todologías extractivistas, que suelen predefinir las técnicas de investigación para posteriormente hacer "encajar» al grupo dentro de ella, hemos optado por invertir los papeles y adaptar estas últimas al grupo. Lejos de anteponer un diseño o un plan de trabajo a priori, hemos intentado acomodarnos al grupo — con sus posibilidades, deseos, disposiciones, tiempos, preferencias, potencialidades y vulnerabilidades-, priorizando aquellos momentos en los que este manifestó la voluntad de participar en el proceso y tomar decisiones. Los interrogantes teóricos-analíticos han ido construyéndose sobre la marcha, no simplemente debido a cierta imprevisibilidad común a toda investigación etnográfica, sino por nuestro intento deliberado de ceder la autoridad etnográfica y construir colectivamente los «qués» y los «cómos» de la investigación. A esto le llamamos pluriversalidad metodológica y consideramos que debería ser un aspecto central de las investigaciones colaborativas, permitiéndonos imaginar otras maneras de escuchar y conversar, con el horizonte de generar "conocimientos en mudanza» (Haber, 2011).

Vayamos ahora a abordar los otros dos ejes mencionados: la co-labor como forma de activar lo(s) común(es) y procesos de subjetivación política $^{11}$.

\subsection{Reivindicando lo(s) común(es) en contextos de cercamiento}

Una investigación centrada en los procesos antes que en los productos, en la colaboración antes que en la competición, abocada al pluriversalismo antes que al individualismo metodológico, se enfrenta a muchas dificultades en el contexto de la Universidad neoliberal y eurocentrada. Por ejemplo, en un texto sobre la investigación-acción también aplicable a nuestro caso, Greenwood señala algunos puntos de tensión inherentes a esta práctica: su vocación holista, anti- o transdisciplinaria, la relevancia epistemológica acordada a los actores locales no académicos, su irreductibilidad a una evaluación desde parámetros cuantitativos y la búsqueda de formas de autoría colectivas (2012: 121-127).

Con el objetivo de ahondar en la discusión, abordaremos las tensiones subyacentes en la relación entre la Universidad neoliberal y los comunes del conocimiento (Hess y Ostrom, 2016) — pues la etnografía colaborativa, como mostraremos, puede ser entendida como una herramienta útil para la institución de común(es) (Cota, Álvarez Veinguer, Olmos Alcaraz, Sebastiani, García Soto y Fernández Alberdi, 2017)—. Para empezar, en la Universidad hay un «cerco» puesto al acceso a los saberes

11. Ambas cuestiones están imbricadas y no son separables salvo en términos abstractos. 
comunes, debido al cuasi monopolio ostentado por empresas como Thomson Reuters o Elsevier en el mercado de las publicaciones científicas. Los investigadores, primero, se ven obligados a publicar los frutos de sus investigaciones - realizadas a menudo en estructuras públicas- en revistas pertenecientes a dichas editoriales, para que posteriormente las universidades vuelvan a adquirir los derechos de acceso a los artículos fruto de su trabajo, generando una segunda transferencia hacia la economía privada y suponiendo gastos exorbitantes para lo público (BermejoBarrera, 2014; Díez Gutiérrez, 2016).

En segundo lugar, el cerco es puesto a la construcción colectiva de saberes: la lógica de investigación imperante, pese a su retórica de apertura, descalifica la participación (como iguales) de actores subalternos en las investigaciones académicas y fomenta una separación entre «sujetos» y «objetos» de estudio. En muchas investigaciones convencionales, las poblaciones estudiadas ni siquiera tienen acceso al conocimiento producido sobre ellas (Manzano-Arrondo, 2015: 209). Lo que es peor: en este escenario, la jerarquía epistemológica entre «saberes expertos»-científicos y supuestamente objetivos- y «saberes no expertos» - populares o pertenecientes a actores con bajo estatus social-, no hace sino profundizarse. En efecto, vista la hegemonía de la «cultura del impacto y de la citación» (Díez Gutiérrez, 2016), el hecho de compartir o producir colectivamente saberes junto con actores "no expertos", pertenecientes a poblaciones marginalizadas o procedentes de mundos sociales no acreditados para producir "plusvalía académica» (por ejemplo, actores sociales sin "poder de citación»), no es rentable para la institución universitaria. Este «fetichismo de los indicadores» ha conllevado la transformación de la actividad científica, puesto que "[e]llos ignoran y destruyen las variedades de formas y prácticas de conocimiento de los distintos campos de estudio. Lo que no es medible y comparable, no cuenta, es un derroche de energía y debería por tanto ser destruido» (Halffman y Radder, 2015: 167, traducción propia). Además, la lógica de los «tiempos rápidos» conlleva que todo lo que se haga más allá de los requerimientos institucionales de «impacto» y «excelencia», todo lo que supere la dimensión de «investigar sobre» para convertirse en «investigar con», al no tener una repercusión directa en el curriculum vitae termine siendo considerado una pérdida de tiempo. Esto tiene efectos concretos sobre la práctica del trabajo de campo: si en un principio toda investigación cualitativa está abierta a escenarios dinámicos y cambiantes que mal se prestan a la redacción de proyectos cerrados o a una implementación «lineal» de los mismos, en el caso de ciertas investigaciones colaborativas e implicadas esta tensión se hace aún mayor, pues los tiempos del proceso de investigación ni siquiera son 
controlados plenamente por el equipo "académico» y a veces dependen de factores totalmente externos a las lógicas de la Universidad (Greenwood, 2012: 127-128). Así, cuanto más coral y colaborativa se haga una etnografía, más duras serán las tensiones y las incompatibilidades con respecto a los tiempos requeridos por la "productividad» académica. Justo en el momento en que más productividad científica se exige a los investigadores, se fomentan prácticas que reducen el tiempo dedicado al trabajo de campo y aumentan el tiempo requerido para la gestión académicaburocrática (Barnés, 2015). Si, por tanto, las lógicas operantes desaniman la producción de saberes comunes junto con actores «marginalizados» o «contrahegemónicos», por otra parte, las universidades no han dejado de firmar acuerdos de cooperación en la investigación con otros tipos de actores, a menudo protegidos por el derecho de autor y en apoyo de intereses privados (casos de grandes empresas) o de poderosas instituciones estatales ${ }^{12}$-estos sí considerados depositarios de los intereses generales de la sociedad-. En términos generales, es posible afirmar que en la Universidad neoliberal la producción de conocimiento es entendida como "[...] un medio de valoración de uno mismo y de su propio 'ridiculum vitae' al margen del valor social del conocimiento como bien común» (Colectivo Indocentia, 2017).

Ante este escenario de tiranía de la individualidad, hace más de una década, Vasco proponía "recoger los conceptos de la vida» (2002) como posible camino para reconectar el trabajo antropológico y las luchas de movimientos indígenas: "[u]na manera contraria de mirar las cosas exige trascender la individualidad, romper con la ficción del sujeto y eliminar la idea de que el sujeto del conocimiento es el etnógrafo. Realmente el sujeto debe ser conjunto, social, integrado por el etnógrafo, que ya porta sobre su espalda el peso de las relaciones de su sociedad que lo determinan, y los indígenas» (Vasco, 2002: 694) ${ }^{13}$. Esta propuesta, junto a otros trabajos realizados en los noventa en Colombia, fue el origen de lo que años más tarde Rappaport (2007) denominaría «etnografía en colaboración», donde el/la antropólogo/a es uno más del grupo, y la colaboración atraviesa todas las fases de la investigación (Arribas Lozano, 2014; Dietz y Álvarez Veinguer, 2014; Rappaport, 2007). Estas contribuciones han propuesto superar el trabajo individual del etnógrafo/a para dotar de centralidad al grupo — un aspecto que, sin duda, entra en directa contradicción con las demandas y lógicas actuales de la Universidad neoliberal-. En términos generales, la colaboración se plantea como una res-

12. Caso de las investigaciones realizadas en colaboración entre Universidad de Granada y Ejército español (Centro Mixto Ugr-Madoc, 2017).

13. Para un análisis del trabajo de Vasco, véase Vasco (2007). 
puesta al problema del «modelo de proyecto individual» (Collier, 2007), que vincula necesariamente la producción académica con elementos individuales del trabajo de campo y de la escritura, se asocia con descripciones densas e interpretaciones virtuosas y elegantes que se consideran la seña de identidad de un buen trabajo. De ahí que se privilegien la experimentación con formas de escritura y estilos de trabajo de campo y se valore el romper con las normas existentes, favoreciendo que las contribuciones asociadas a determinados autores adopten frecuentemente la forma de conceptos «marcas» (Collier, 2007: 55). Ante este modelo vanguardista, pensamos que superar y trascender la sobrevaloración de la figura individual del/a etnógrafo/a sin duda puede contribuir a activar un intercambio y una ecología de saberes para aprender nuevas formas de habitar las producciones de sentido que estén atravesadas por la lógica instituyente de lo común. Se trata, entonces, de apostar por la figura del/a académico/a anónimo/a que sabe diluirse junto al grupo, capaz de poner sus conocimientos a disposición, sin buscar reconocimientos y réditos sino para construir, dialogar, desmontar y transformar de manera colectiva, construyendo espacios donde dispongamos de tiempo para escucharnos, contarnos y compartirnos. Desde nuestra coinvestigación, un ejemplo de ello es el proceso que nos ha llevado desde la implementación inicial de unos «dispositivos de escucha» a la realización de un proyecto de narrativa audiovisual transmedia ${ }^{14}$ en el que estamos actualmente trabajando. De manera resumida: en un primer momento, impulsamos la realización de «grupos de debate» junto a una asamblea de Stop Desahucios — la del barrio del Zaidín - y de entrevistas en la otra — la Asamblea Centro ${ }^{15}$ Por limitaciones de espacio no podemos detenernos a analizar el contenido de los diferentes "grupos de debate», pero sí la forma de estos: antes de comenzar a debatir, alcanzamos colectivamente algunos acuerdos de funcionamiento que se recogieron y se colgaron en la sala de reuniones. Dichos acuerdos se resumían en: 1) estamos aquí para debatir, analizar y reflexionar sobre nuestros discursos y prácticas para fortalecer nuestro movimiento; 2 ) es nuestra propia voz y nuestras experiencias vividas lo que tiene valor. Hablar con respeto y entender que hay otro punto de vista; 3) dejar el móvil en silencio. Entrar y salir sin molestar; 4) sentirnos

14. El proyecto transmedia comenzó en Octubre de 2017, y se encuentra en sus fases iniciales cuando escribimos este artículo.

15. Desde noviembre de 2015 hemos estado participando en dos asambleas del movimiento en la ciudad de Granada: la primera está ubicada en la zona sur, en el barrio popular y obrero del Zaidín, mientras que la segunda, aun encontrándose en la zona Centro, incluye en su área de intervención otras zonas populares o empobrecidas de la ciudad. Para más informaciones: https://afectadosporlahipotecagranada.com. 
lo más libres posible para expresar nuestra opinión. Escucharnos los/as unos/as a los/as otros/as.

Acordar las normas básicas de funcionamiento (coproducción de normas) antes de comenzar a debatir, podría parecer un gesto insignificante y puramente organizativo. Sin embargo, es uno de los elementos centrales para interiorizar y compartir las «reglas de juego» que estábamos estableciendo, y consecuentemente haciéndolas propias para gestionar el espacio de escucha que queríamos construir (práctica bastante habitual dentro de los espacios y colectivos asamblearios y, sin embargo, poco frecuente en la mayoría de investigaciones etnográficas). Es una manera de activar procesos de agenciamiento donde el grupo asume e interioriza la responsabilidad compartida de la construcción de los espacios de escucha. Frente a las técnicas más tradicionales donde se impone la forma, el contenido y los tiempos, hemos entendido que los «grupos de debate», así como cualquier otro dispositivo de producción de sentido que se acuerde desplegar, deben operar desde los principios de la escucha, horizontalidad y el máximo respeto. La versatilidad y flexibilidad de adaptarse al grupo, y no al revés, nos ha permitido responder y adaptarnos a las condiciones de cada grupo. El objetivo de estas «técnicas», sin embargo, no era el de generar conocimiento alrededor de dilemas teóricos previamente definidos por el «equipo investigador»: por el contrario, se trataba de utilizarlas «instrumentalmente» para hacer emerger los «qués» de una manera coral, a partir de las necesidades sentidas por el grupo. Las personas vinculadas con la universidad no éramos exclusivamente "observadoras» del grupo, o moderadoras en el sentido de «hacer hablar», ni expertas en el tema tal como se presupone en otras técnicas de investigación. Fuimos participando al igual que el resto de los miembros de los grupos en los debates cuando considerábamos que teníamos algo que aportar y compartir. Solo posteriormente, y en función de lo que había emergido, elaboramos la propuesta de construir una pluralidad de narraciones audiovisuales (proyecto transmedia) para dar a conocer la realidad de Stop Desahucios Granada-15M, potenciar su perfil público e impulsar su capacidad para tejer alianzas con otros actores. Pero todo este proceso fue construido y negociado continua y colectivamente. La construcción de este proyecto transmedia no solo desborda la lógica individual de investigación, sino que resulta hasta contraproducente desde una óptica «productivista»y de «tiempos rápidos». Podríamos habernos dedicado únicamente a analizar y publicar fragmentos «extraídos» de nuestros grupos de debate y entrevistas, alcanzando un mayor impacto académico, pero eso habría supuesto establecer una relación entre "equipo de investigación» y «grupo activista» en términos de «sujeto» vs. «objeto». En 
cambio, optamos por usar los materiales producidos de manera «interna», como herramientas para arrancar un proceso colectivo, definir intereses comunes y responder a necesidades sentidas y planteadas por el propio grupo. Este es un ejemplo de cómo, frente a las técnicas extractivistas que vampirizan los saberes de los sujetos que participan en las investigaciones, es posible plantear otros objetivos y propósitos: no extraer y desposeer, sino dar, compartir y reaprender. Desde este punto de vista, esta co-labor nos está permitiendo trascender la dimensión individual, activando la producción de sentido de forma colectiva a través de la construcción de lo(s) común(es) (Cota et.al., 2017) ${ }^{16}$.

\subsection{Procesos de subjetivación política en contextos de despolitización}

Algunos estudiosos han hablado de «ciencia neoliberal» (Lander, 2005) o "conocimiento neoliberalizado» (Brown, 2011) para indicar el tipo de saberes impulsados por el actual modelo de Universidad. En particular, se han resaltado estos aspectos:

- Se producen saberes «inmediatos» y fácilmente trasladables a contextos de aplicación tecnológica (Escobar, 2007: 53-55). Saberes justin-time y ready-for-use cuya naturaleza fragmentada, especializada y compartimentada no suele tener utilidad más allá del ámbito restringido para el cual son creados. Es un paradigma no solamente "cortoplacista», sino también "tecnocrático", que dificulta considerablemente la financiación de aquellas investigaciones más comprometidas socialmente, cuyos resultados no son visibles a corto plazo, ni son fácilmente valorables mediante estándares «objetivos».

- Prevalecen paradigmas neopositivistas, cientificistas y cuantitativos (Ceglowski, Bacigalupa y Peck, 2011). Se producen saberes despolitizados y pretendidamente "neutros» que, no por ocultar su lugar de enunciación (sus "porqués» y "para qués»), dejan de tenerlo. Prevalecen estudios hechos en universidades del «Norte» global (Galcerán, 2010: 15), validados desde criterios autorreferenciales de excelencia (Lander, 2005: 50) y casi siempre comunicados en inglés (Díez Gutiérrez, 2016; Galcerán, 2010: 15). Y todo ello apuntala aún más la colonialidad del saber ya presente en la Universidad tradicional.

16. Por problemas de espacio en este artículo no podremos explicar en detalle el proceso colaborativo que estamos realizando. Aconsejamos leer el trabajo de Ariana Sánchez Cota y Antonia Olmos Alcaraz en este mismo monográfico, porque forman parte del mismo equipo y desarrollan en mayor profundidad algunas de las cuestiones que aquí son enunciadas esquemáticamente o de una manera que podría parecer "programática». 
- Sin debatir ahora la separación moderna entre ciencias «duras» y ciencias sociales y humanas (Wallerstein, 1997: 112), indudablemente las primeras —en particular las «ciencias de la vida»- se encuentran claramente privilegiadas (Escobar, 2007: 55; Galcerán, 2010: 16); las segundas, al no ser tan fácilmente rentabilizables, en algunas de sus vertientes más antipositivistas llegan incluso a ser definidas como «no científicas» (Brown, 2011: 117).

- Se priorizan los aspectos procedimentales -mediciones, indicadores, evaluaciones de impacto- (Manzano-Arrondo, 2015: 202) sin que medie una reflexión sobre la no neutralidad de estos y sus implicaciones. Estos dispositivos terminan influyendo en la elección de los temas de investigación, las categorías de análisis, las metodologías, los formatos de divulgación —anteponiendo el formato artículo científico a los demás (Manzano-Arrondo, 2015: 206)—, los estilos de escritura y hasta los lenguajes para la redacción de proyectos y textos científicos ${ }^{17}$.

Ahora bien, lo que nos parece interesante de nuestra experiencia de co-labor no son tanto los objetivos políticos perseguidos — que compartimos y promovemos en tanto que activistas-, sino los procedimientos metodológicos desplegados, en la medida en que ayudan a activar procesos de (re)politización colectivos. Frente a la instaurada despolitización dentro y fuera de la universidad, en los grupos de debate realizados, las/ los compañeras/os compartían que la primera vez que asistían a una asamblea de Stop Desahucios, los sentimientos más recurrentes eran el miedo, la soledad, el abandono y la vulnerabilidad. La frustración ante el fracaso, el complejo por la incapacidad de hacer frente a los pagos, la vergüenza y la presión social por el "qué dirán» se repetían en las primeras experiencias ante la amenaza del desahucio. Sin embargo, el encontrarse con otras personas con trayectorias vitales compartidas, permitió activar procesos de agenciamiento donde el individuo que inicialmente solo quería no perder su casa se veía atravesado y sobrepasado por una inteligencia colectiva (un hacer común) que desbordaría y revertiría en todas las acciones diarias del movimiento, mediante los roces, las vivencias y los afectos que

17. Caso de la antropóloga italiana Roberta Chiroli, cuya tesis doctoral es una etnografía del movimiento "No Tav» que se opone a los trenes de Alta Velocidad en Val di Susa, en Piamonte (Chiroli, 2017). Roberta fue imputada y finalmente condenada a dos meses de cárcel, por haber realizado observación participante durante una manifestación terminada con desórdenes. Aunque en ningún momento estuviera implicada en los sucesos, el juez la consideró «moralmente responsable» por haber descrito esos acontecimientos recurriendo a la primera persona del plural (Giambartolomei, 2016). El que un juez pueda erigirse en autoridad epistemológica y emitir condenas en función de su propia noción de investigación nos resulta muy preocupante y apunta a la emergencia de un paradigma de «investigación obediente» (Colectivo Indocentia, 2016). 
construyen la coimplicación. No solo se aprende a gestionar y resolver los problemas concretos de cómo enfrentarse a una situación de soledad y abandono ante la imposibilidad de hacer frente a una hipoteca adquirida o no poder pagar el alquiler: paulatinamente, el día a día se politiza porque lo primero que se pierde es el miedo, al sentirse acompañado en el camino. Sin querer idealizar el proceso de agenciamiento, que evidentemente también tiene sus tensiones y fisuras, el grupo, de forma colectiva, se despliega más allá de las individualidades. Se aprende a tomar la palabra y a negociar con los bancos, cada cual se hace experto en formas de proceder, surfeando las trampas del sistema y convirtiéndose en experto/a, reconocido/a y valorado/a dentro del grupo. Las personas devienen en máquinas de deseos que toman las calles, cantan, bailan y pelean en una cotidianidad donde la política y su gramática ya es otra: no solo se cuidan y luchan para no perder sus casas, sino que sostienen un movimiento que reivindica el derecho de toda persona a una vivienda digna. El cambio es sustancial: del motivo individual se pasa a la causa colectiva. Se produce una reapropiación del espacio público, se aprende a rellenar escritos, a pelear en los juzgados y a conversar de igual a igual con abogados/as y procuradoras/es. Un desborde creativo (que no opera en las formas tradicionales de hacer de los partidos de izquierdas vanguardistas), que traspasa y supera la política formal porque las/os protagonistas, los mensajes y las formas son otras. Se habita una política de los vínculos (Segato, 2016) atravesada por las dimensiones relacionales, comunicativas y de cuidados. Se generan otros lenguajes y formas de relacionarse que antes no se practicaban y cuya existencia se desconocía. Es un hacer-habitar, que antes no les interpelaba y no formaba parte de su día a día. La crisis y la experiencia de gestionar colectivamente los problemas relativos a la vivienda en Stop Desahucios, han generado unas dinámicas de agenciamiento que devienen en un proceso de subjetivación política (Tassin, 2012). En el nuevo dialogo de sujetos a sujetos, son actores centrales que redefinen las representaciones de lo posible. Se hacen cosas que no se sabía que se podía/sabía hacer, se desestabilizan las jerarquías de poder al definir y reconocer quién puede o no hacer investigación, quién puede o no producir conocimiento. En otras palabras, no solo se convierten en interlocutores/as válidos/as para los bancos — porque aquí no hay intermediarios/as ni agentes expertos y especializados con saberes técnicos ajenos al movimiento-, sino que también se colocan como actores protagonistas para producir conocimiento desde y sobre su movimiento. Pero algo parecido nos pasa a los miembros del «equipo de investigación»: en el emocionante camino de aprender y desaprender mediante la coinvestigación, ya no somos las mismas que hace dos años. Los miembros de la investi- 
gación participamos de forma colectiva en la producción de sentido y en el cuestionamiento de la autoridad etnográfica y de su lugar privilegiado de enunciación, y muchos papeles que previamente habíamos aprendido se difuminan o incluso desaparecen. Entonces, la acción de pensarnos, conocernos, vivir y compartir las emergencias diarias del movimiento mientras construimos la investigación, también es un proceso de subjetivación política, por medio del cual devenimos en subjetividades otras a lo largo del proceso de coinvestigación.

\section{Algunas líneas de cierre...}

El compromiso es la disposición de dejarse comprometer, a ser puestos en un compromiso por un problema no previsto que nos asalta y nos interpela. El compromiso, así, es a la vez activo y pasivo, decidido y receptivo, libre y coaccionado (Garcés, 2011).

En este artículo, primero, hemos repasado las condiciones de la institución académica contemporánea, definiéndola una Universidad neoliberal y eurocentrada. Desde nuestro contexto específico, hemos resaltado que las tendencias actuales transforman los tiempos y las subjetividades de la investigación, supeditándolos a criterios de productividad e individualismo, generando saberes despolitizados y precarizando las condiciones de trabajo y de vida. Posteriormente, hemos conceptualizado la etnografía colaborativa como un intento por decolonizar las prácticas de investigación, resaltando su potencial para activar lógicas instituyentes del común y fomentar procesos de subjetivación política, así como subrayando su capacidad para poner en valor los cuidados / lo emocional / lo afectivo y establecer dispositivos de escucha que indisciplinen las metodologías, generando procesos de investigación más horizontales. Hemos discutido particularmente los primeros dos aspectos, aportando ejemplos de nuestra coinvestigación y poniéndolos en diálogo con los escenarios supuestos por nuestra pertenencia a la institución académica, resaltando tensiones y dificultades. A este respecto, insistimos: aunque no tengamos la pretensión de aportar «soluciones» a gran escala, consideramos fundamental seguir preguntándonos — cada cual desde los contextos específicos de su quehacer académico- por posibles maneras de cuestionar las tendencias más individualistas y despolitizadoras de la Universidad neoliberal. Añadimos que este cuestionamento, a nuestro modo de ver, debería darse no solo a nivel teórico y discursivo, sino también en el terreno de las elecciones metodológicas que afectan a la cotidianidad del trabajo de campo. Es 
cierto que el contexto general es tendencialmente negativo, pero también existen importantes márgenes de acción aprovechables. En efecto, la Universidad actual no deja de caracterizarse por la copresencia de discursos, lógicas y prácticas ambivalentes, incluso «esquizofrénicas» (Shore, 2010: 21). Es por tanto un espacio contradictorio, donde posibilidades alternativas y potenciales líneas de fuga puede que se hayan reducido, pero siguen existiendo. Por ejemplo, con respecto de los comunes, asistimos al protagonismo de algunas universidades que fomentan el uso del software libre - aunque sea para «racionalizar los costes»- y alientan a sus investigadores a poner en código abierto las versiones pre-print de sus artículos - aunque sea para aumentar las probabilidades de citación y de subir en los rankings internacionales- (Hess y Ostrom, 2016: 34). En el plano de la acción colectiva, aparecen críticas cada vez más consistentes a este modelo de Universidad - caso de las propuestas que abogan por una Universidad «lenta», expresión basada en el título de un volumen recién publicado (Berg y Seeber, 2016)—. Ante la precariedad, se están dando intentos de autoorganización por parte del personal investigador —caso del encuentro "PrecAnthro», organizado durante el Congreso EASA de Milán (2016), que ha visto la participación de más de ciento cincuenta antropólogos/as y ha sentado las bases para la construcción de una red transnacional (AllegraLab, 2016)—. Finalmente, en el interior de la práctica etnográfica, desde distintas localizaciones emergen experiencias comprometidas que cuestionan y desafían la narración eurocéntrica de la modernidad, así como las lógicas extractivistas de sus saberes. Tal como recoge el presente monográfico, resuenan múltiples experiencias desde diferentes coordenadas espaciotemporales que nos permiten seguir rastreando maneras diferentes de hacer/habitar dentro y contra la Universidad neoliberal y eurocentrada.

\section{Referencias}

AAVV. (2015). Prácticas otras de conocimiento(s): entre crisis, entre guerras. San Cristóbal de las Casas: Cooperativa Editorial Retos.

Alinovi, M. (2014). Excelencia, relevancia y política científica. SEBBM, 180: 10-12.

AllegraLab (2016). Towards a Transnational Anthropology Union \#UniversityCrisis. En http://allegralabzoratory.net/towards-a-transnational-anthropology-union-universitycri sis/. Accedido el 11 de noviembre de 2017.

Alonso, L. y Fernández Rodríguez, C. (2016). La burocracia neoliberal y las nuevas funciones de las normas. Encrucijadas, 12: 1-26. 
Arribas Lozano, A. (2014). Formas de Hacer. Experimentación y prácticas emergentes en los movimientos sociales. Una etnografía de las Oficinas de Derechos Sociales. Tesis Doctoral no publicada, Granada, Universidad de Granada.

Barnés, H. (2015). Esto es a lo que dedican de verdad su tiempo los profesores, aunque no quieran. El Confidencial. En http://www.elconfidencial.com/alma-corazon-vida/2015-05-29/ esto-es-a-lo-que-dedican-su-tiempo-los-profesores-aunque-no-lo-quieran_859151/. Accedido el 11 de noviembre de 2017.

Behar, R. (1996). The vulnerable observer: anthropology that breaks your heart. Boston: Beacon press.

Berardi, F. (2003). La fábrica de la infelicidad. Nuevas formas de trabajo y movimiento global. Madrid: Traficantes de Sueños.

Berg, M. y Seeber, B. (2016). The Slow Professor: Challenging the Culture of Speed in the Academy. University of Toronto.

Bermejo-Barrera, J. (2014). La nueva servidumbre: microeconomía de las publicaciones científicas. USC fírgoa. En http://firgoa.usc.es/drupal/node/51197. Accedido el 11 de noviembre de 2017.

Blommaert, J. (2015). Rationalizing the unreasonable: there are no good academics in the EU. Ctrl+Alt+Dem Research on alternative democratic life in Europe. En https://alter native-democracy-research.org/2015/06/10/rationalizing-the-unreasonable-there-are-nogood-academics-in-the-eu/. Accedido el 11 de noviembre de 2017.

Braidotti, R. (2004). Feminismo, diferencia sexual y subjetividad nómada. Barcelona: Gedisa. Brown, W. (2011). Neoliberalized knowledge. History of the Present, 1(1): 113-129.

Castillo, J. y Moré, P. (2017). La Universidad sin futuro. Precariedad e incertidumbre del trabajo universitario. Madrid: Universidad Complutense de Madrid.

Castro-Gómez, S. (2005). La Hybris del Punto Cero: ciencia, raza e Ilustración en la Nueva Granada (1750-1816). Bogotá: Pontificia Universidad Javeriana.

Castro-Gómez, S. y Grosfoguel, R. (2007). El giro decolonial. Reflexiones para una diversidad epistémica más allá del capitalismo global. Bogotá: Siglo del Hombre.

Ceglowski, D.; Bacigalupa, C. y Peck, E. (2011). Aced Out: Censorship of Qualitative Research in the Age of "Scientifically Based Research". Qualitative Inquiry, 17(8): 679686.

Centro Mixto Ugr-Madoc (2017). Plan anual de colaboración Ugr-Madoc 2017. En http://cemixugrmadoc.ugr.es/pages/actividades/pac2017/. Accedido el 11 de noviembre de 2017.

Chiroli, R. (2017). Ora e sempre no TAV. Pratiche e identità del movimento valsusino contro l'Alta Velocità. Milano: Mimesis.

Chomsky, N. (2013). El trabajo académico, el asalto neoliberal a las universidades y cómo debería ser la educación superior. Bajo el Volcán, 13(21): 121-134.

Colectivo Indocentia (2017). Manifiesto universitario del colectivo Indocentia: el programa Docentia o la corrupción de la docencia. En https:/www.uv.es/cgt/COMUNITAT_ UNIVERSITARIA/PDI/Indocentia_cas.pdf. Accedido el 11 de noviembre de 2017.

Colectivo Indocentia (2016). Disciplinar la investigación, devaluar la docencia: cuando la Universidad se vuelve empresa. Entrevista por Amador Fernández-Savater. Eldiario.es. 
En http://www.eldiario.es/interferencias/Disciplinar-investigacion-devaluar-docenciaUniversidad_6_486161402.html. Accedido el 11 de noviembre de 2017.

Collier, J. (2007). The Collaboratory Form in Contemporary Anthropology. En Concept Work and Collaboration in the Anthropology of the Contemporary. T. Rees, Comp. ARC Exchange, 1: 54-61.

Consejo Europeo (2000). Consejo europeo de Lisboa, 23 y 24 de marzo de 2000. Conclusiones de la presidencia. http://www.europarl.europa.eu/summits/lis1_es.htm\#b. Accedido el 11 de noviembre de 2017.

Cota, A. S.; Álvarez Veinguer, A.; Olmos Alcaraz, A.; Sebastiani, L.; García Soto, R. y Fernández Alberdi, B. (2017). La construcción de comunes en la lucha por la vivienda: etnografía colaborativa junto al colectivo Stop Desahucios 15M Granada y la PAH de Barcelona. En Rebeldías en Común. A. Calle, Ed. Madrid: Libros en Acción.

Díez Gutiérrez, E. (2016). Más allá del negocio de los sexenios universitarios. Nuevatribuna. es. En http://www.nuevatribuna.es/articulo/sociedad/mas-alla-negocio-sexenios-univer sitarios/20161005102328132368.html. Accedido el 11 de noviembre de 2017.

Dietz, G. y Álvarez Veinguer, A. (2014). Reflexividad, interpretación y colaboración en etnografía: un ejemplo desde la antropología de la educación. En La etnografía y el trabajo de campo en las ciencias sociales. C. Oehmichen, Ed. México D.F.: Universidad Autónoma de México.

Eizagirre, A. (2016). La constitución de las políticas de investigación e innovación responsables: tensiones en la instrumentalización y la regulación. Política y Sociedad, 53(3): 815-836.

Escobar, C. (2007). Universidad, conocimiento y subjetividad. Relaciones de saber/poder en la academia contemporánea. Nómadas, 27: 48-61.

Esteban, M. (2015). La reformulación de la política, el activismo y la etnografía. Esbozo de una antropología somática y vulnerable. Ankulegi, 19: 75-93.

Esteban, M (2004). Antropología del cuerpo: géneros, itinerarios corporales, identidad y cambio. Barcelona: Bellaterra.

Fernández-Savater, A. (2008). Politizar el sufrimiento. Minerva, 7: 111-112.

Ferreiro Baamonde, X. (2010). Mercantilización y precarización del conocimiento: el proceso de Bolonia. En La Universidad en conflicto. Capturas y fugas en el mercado global del saber. Edu-Factory y Universidad Nómada, Comps. Madrid: Traficantes de Sueños.

Galcerán, M. (2010). La educación universitaria en el centro del conflicto. En La Universidad en conflicto. Capturas y fugas en el mercado global del saber. Edu-Factory y Universidad Nómada, Comps. Madrid: Traficantes de Sueños.

Garcés, M. (2011). Renovar el compromiso. Espai en Blanc, 9-11. En http://espaienblanc. net/?page_id=621. Accedido el 11 de noviembre de 2017.

Giambartolomei, A. (2016). No Tav, Roberta Chiroli e la tesi di laurea «moralmente complice». Condannata a 2 mesi. Il Fatto Quotidiano. En http://www.ilfattoquotidiano.it/ premium/articoli/i-no-tav-roberta-e-la-tesi-moralmente-complice/. Accedido el 11 de noviembre de 2017.

Gill, R. (2015). Rompiendo el silencio. Las heridas ocultas de la universidad neoliberal. Arxius, 32: 45-58. 
Gómez, L. y Jódar, F. (2013). Ética y política en la universidad española: la evaluación de la investigación como tecnología de la subjetividad. Athenea Digital, 13(1): 81-98.

Greenwood, D. (2012). Doing and learning action research in the neo-liberal world of contemporary higher education. Action Research, 10(2): 115-132.

Gregorio Gil, C. (2006). Contribuciones feministas a problemas epistemológicos de la disciplina antropológica: representación y relaciones de poder. AIBR. Revista de Antropología Iberoamericana, 1(1): 22-39.

Grosfoguel, R. (2016). Del «extractivismo económico» al «extractivismo epistémico» y al «extractivismo ontológico»: una forma destructiva de conocer, ser y estar en el mundo. Tabula Rasa, 24: 123-124.

Haber, A. (2011). Nometodología Payanesa: Notas de Metodología. Indisciplinada. Revista de Antropologia, 23(1): 9-49.

Hale, C. (2011). Entre el mapeo participativo y la "geopiratería»: las contradicciones (a veces constructivas) de la antropología comprometida. En Conocimientos y prácticas políticas: reflexiones desde nuestras prácticas de conocimiento situado (Tomo II). X. Leyva et al., Ed. Chiapas: CIESAS.

Halffman, W. y Radder, H. (2015). The Academic Manifesto: From an Occupied to a Public University. Minerva, 53(2): 165-187.

Haraway, D. (1991). Simians, Cyborgs and Women. New York: Routledge.

Hess, C. y Ostrom, E. (2016). Los bienes comunes del conocimiento. Quito: Instituto de Altos Estudios Nacionales de Ecuador.

Irigoyen, J. (2011). La refundación de la evaluación. Laberinto, 33: 35-44.

Lander, E. (2005). La ciencia neoliberal. Revista Venezolana de Economía y Ciencias Sociales, 11(2): 35-69.

Lander, E. (2000). La colonialidad del saber: eurocentrismo y ciencias sociales. Perspectivas latinoamericanas. Buenos Aires: CLACSO.

Lara, A. y Álvarez, A. (2009). Gubernamentalidad, precarización y nueva servidumbre. Agenciamientos a partir de los circuitos de producción mercantil de cuidados y subjetividades. Sociología del Trabajo, 66: 107-131.

Leyva, X.; Burguete, A. y Speed, S. (2008). Gobernar (en) la diversidad: experiencias indígenas desde América Latina. Hacia la investigación de co-labor. México D.F.: CIESAS.

Lugones, M. (2008). Colonialidad y género. Tabula Rasa, 9: 73-101.

Lutz, C. y Abu-Lughod, L. (1990). Language and the politics of emotion. Cambridge: Cambridge University Press.

Manzano-Arrondo, V. (2015). Academia, Evaluación y Poder. Revista de la Asociación de Sociología de la Educación, 8(2): 198-223.

Migliaccio, F. (2016). La fabbrica dei dottorandi. Gli asini. En https://gliasinirivista.org/ lafabbrica-dei-dottorandi/. Accedido el 11 de noviembre de 2017.

Montalba Ocaña, C. (2015). El sueño de la excelencia. Desvelarlo, desvelar-nos. Arxius, 32: 83-98.

Olmos Alcaraz, Antonia; Cota, Ariana S.; Álvarez Veinguer, Aurora; Sebastiani, Luca (2018). Etnografía con los movimientos de lucha por el derecho a la vivienda en el sur de 
Europa: retos metodológicos en la investigación colaborativa para la acción social. Universitas Humanística, 86: 139-166.

Pérez Rastrilla, L. (2017). Profesores a precio de ganas. Fuegoamigo. En http://fuegoamigo. es.linkis.com/MwOWi. Accedido el 11 de noviembre de 2017.

Rappaport, J. (2007). Más allá de la escritura: la epistemología de la etnografía en colaboración. Revista Colombiana de Antropología, 43: 197-229.

Rich, A. (1986). Blood, Bread and Poetry. Selected Prose 1979-1985. London: Virago.

Risager, B. y Thorup, M. (2016). Protesting the Neoliberal University: The Danish Student Movement "A Different University". Interface: a journal for and about social movements, 8(1): 7-33.

Santos, B. de S. (2009). Una epistemología del Sur. La reinvención del conocimiento y la emancipación social. México: Siglo XXI.

Scheper-Hughes, N. y Lock, M. (1987). The Mindful Body: A Prolegomenon to Future Work in Medical Anthropology. Medical Anthropology Quarterly New Series,1(1): 6-41.

Segato, R. (2016). La guerra contra las mujeres. Madrid: Traficantes de Sueños.

Serrano, A. y Crespo, E. (2002). El discurso de la Unión Europea sobre la sociedad del Conocimiento. Reis, 97: 189-207.

Shore, C. (2010). Beyond the multiversity: neoliberalism and the rise of the schizophrenic university. Social Anthropology, 18(1): 15-29.

Shore, C. y Wright, S. (2015). Governing by numbers: audit culture, rankings and the new world order. Social Anthropology, 23(1): 22-28.

Smith, L. (1999). Decolonizing Methodologies. New Zealand: University of Otago.

Stavenhagen, R. (1971). Decolonizing applied social sciences. Human Organization, 30(4): 33-44.

Tassin, E. (2012). De la subjetivación política. Althusser/Rancière/Foucault/Arendt/Deleuze. Revista de Estudios Sociales, 43: 36-49.

Vasco, L. (2007). Así es mi método en etnografía. Tabula Rasa, 6: 19-52.

Vasco, L. (2002) Entre Selva y paramo. Viviendo y pensando la lucha india. Bogotá: Instituto Colombiano de Antropología e Historia.

Wallerstein, I. (1997). El eurocentrismo y sus avatares: los dilemas de las ciencias sociales. New Left Review, 0: 97-113.

Walsh, C.; Schiwy, F. y Castro-Gómez, S. (2002). Indisciplinar las ciencias sociales. Geopoliticas del conocimiento y colonialidad del poder. Perspectivas desde lo Andino. Quito: Universidad Andina Simón Bolívar/Abya Yala.

Wright, S. y Rabo, A. (2010). Introduction: Anthropologies of university reform. Social Anthropology, 18(1): 1-14. 
